\title{
Generalized Almost Distributive Fuzzy Lattices
}

\author{
Berhanu Assaye Alaba and Yohannes Gedamu Wondifraw
}

\begin{abstract}
In this paper we introduce the concept of a Generalized almost distributive fuzzy lattices (GADFLs) as a generalization of an Almost distributive fuzzy lattices(ADFLs). Again, we also show that a necessary and sufficient conditions for a GADFLs to become a distributive fuzzy lattices and a GADFLs to become an ADFLs.
\end{abstract}

Index Terms-Almost distributive fuzzy lattice, distributive fuzzy lattice, fuzzy poset, generalized almost distributive fuzzy lattice, generalized almost distributive lattice.

\section{INTRODUCTION}

$\mathbf{T}$ HE concept of a Generalized Almost Distributive Lattice (GADL) was introduced by Rao, Ravi Kumar and Rafi [1] as a generalization of an Almost Distributive Lattice (ADL) [2] which was a common abstraction of almost all the existing ring theoretic generalization of a Boolean algebra on one hand and distributive lattice on the other. The class of GADLs inherit almost all the properties of distributive lattice except possibly the commutativity of $\wedge, \vee$, the right distributivity of either of the operations $\vee$ or $\wedge$ over the other. The class of GADLs include the class of ADLs properly and retain many important properties of ADLs. On the other hand, L. A. Zadeh [3] in 1965 introduced the notion of fuzzy set to describe vagueness mathematically in its very abstractness and tried to solve such problems by assigning to each possible individual in the universe of discourse a value representing its grade of membership in the fuzzy set. Again in 1971, Zadeh [4] defined a fuzzy ordering as a generalization of the concept of ordering, that is, a fuzzy ordering is a fuzzy relation that is transitive. In particular, a fuzzy partial ordering is a fuzzy ordering that is reflexive and antisymmetric. In 1994, Ajmal and Thomas [5] defined a fuzzy lattice as a fuzzy algebra and characterized fuzzy sublattices. In 2009, Chon [6], considering the notion of fuzzy order of Zadeh, introduced a new notion of fuzzy lattice and studied the level sets of fuzzy lattices. He also introduced the notions of distributive and modular fuzzy lattices and considered some basic properties of fuzzy lattices.

In this paper, we introduce the concept of Generalized Almost Distributive Fuzzy Lattices (GADFLs) as a generalization of an Almost Distributive Fuzzy Lattices (ADFLs) using the fuzzy partial order relation and fuzzy lattice defined by Chon [6].

\section{PRELIMINARIES}

First, we recall certain definitions and properties of Generallized Almost Distributtive Lattices that are required in this paper.

Manuscript received January 11, 2017; accepted May 18, 2017.

The authors are with the Department of Mathematics, College of Science, Bahir Dar University, Bahir Dar, Ethiopia. Email: berhanu_assaye@yahoo.com, yohannesg27@gmail.com
Definition 1 ([1]): An algebra $(L, \vee, \wedge)$ of type $(2,2)$ is called a Generalized Almost Distributive Lattice if it satisfies the following axioms:

$(A s \wedge)(x \wedge y) \wedge z=x \wedge(y \wedge z)$

$(\mathrm{LD} \wedge) \quad x \wedge(y \vee z)=(x \wedge y) \vee(x \wedge z)$,

$\mathrm{LD} \vee) x \vee(y \wedge z)=(x \vee y) \wedge(x \vee z)$,

$\left(A_{1}\right) x \wedge(x \vee y)=x$,

$\left(A_{2}\right)(x \vee y) \wedge x=x$,

$\left(A_{3}\right)(x \wedge y) \vee y=y$, for all $x, y, z \in L$.

Lemma 2 ([1]): For any $a \in L$,

(1) $a \vee a=a$,

(2) $a \wedge a=a$.

In addition to the 3 absorption laws $A_{1}, A_{2}, A_{3}$ given in definition 2.1, we also get the following:

Lemma 3 ([1]): For any $a, b \in L$,

$\left(A_{4}\right) \quad a \vee(a \wedge b)=a$,

$\left(A_{5}\right) \quad a \vee(b \wedge a)=a$.

Corollary 4 ([1]): For any $a, b \in L$, we have

(1) $a \vee(a \vee b)=a \vee b$,

(2) $(a \vee b) \vee a=a \vee b$,

(3) $a \wedge(b \wedge a)=b \wedge a$.

Definition 5 ([1]): For any $a, b \in L$ we say that $a$ is less than or equal to $b$ and write $a \leq b$, if $a \wedge b=a$ or equivalently, $a \vee b=b$.

Lemma 6 ([1]): For any $a, b \in L$,

(1) $a \wedge b \leq b$,

(2) $a \leq a \vee b$.

Lemma 7 ([1]): If $a, b \in L$ such that $a \leq b$ then,

(1) $a \wedge b=b \wedge a$,

(2) $a \wedge(b \vee a)=a$,

(3) $a \vee b=b \vee a$

Lemma 8 ([1]): For any $a, b, c \in R, a \wedge b \wedge c=b \wedge a \wedge c$ Next, we give some properties and definitions of Fuzzy Partial Order Relation, Fuzzy Lattice and Fuzzy Distributive Lattice.

Definition 9 ([6]): Let $X$ be a set. A function $A: X \times X \longrightarrow$ $[0,1]$ is called a fuzzy relation in $X$. The fuzzy relation $A$ in $X$ is reflexive if and only if $A(x, x)=1$ for all $x \in X, A$ is transitive if and only if $A(x, z) \geq \sup _{y \in X} \min (A(x, y), A(y, z))$, and $A$ is antisymmetric if and only if $A(x, y)>0$ and $A(y, x)>0$ implies $x=y$. A fuzzy relation $A$ is fuzzy partial order relation if $A$ is reflexive, antisymmetric and transitive. A fuzzy partial order relation $A$ is a fuzzy total order relation if and only if $A(x, y)>0$ or $A(y, x)>0$ for all $x, y \in R$. If $A$ is a fuzzy partial order relation in a set $X$, then $(X, A)$ is called a fuzzy partially ordered set or a fuzzy poset. If $B$ is a fuzzy total order relation in a set $X$, then $(X, B)$ is called a fuzzy totally ordered set or a fuzzy chain.

Now we define a fuzzy lattice as a fuzzy partial order relation. 
Definition 10 ([6]): Let $(X, A)$ be a fuzzy poset and let $B \subseteq X$. An element $u \in X$ is said to be an upper bound for a subset $B$ iff $A(b, u)>0$ for all $b \in B$. An upper bound $u_{0}$ for $B$ is the least upper bound of $B$ if and only if $A\left(u_{0}, u\right)>0$ for every upper bound $u$ for $B$. An element $v \in X$ is said to be a lower bound for a subset $B$ iff $A(v, b)>0$ for all $b \in B$. A lower bound $v_{0}$ for $B$ is the greatest lower bound of $B$ if and only if $A\left(v, v_{0}\right)>0$ for every lower bound $v$ for $B$.

We denote the least upper bound of the set $\{x, y\}$ by $x \vee y$ and denote the greatest lower bound of the set $\{x, y\}$ by $x \wedge y$.

Definition $11([6])$ : Let $(X, A)$ be a fuzzy poset. $(X, A)$ is a fuzzy lattice if and only if $x \vee y$ and $x \wedge y$ exist for all $x, y \in X$.

Definition 12 ([6]): Let $(X, A)$ be a fuzzy lattice. $(X, A)$ is distributive if and only if $x \wedge(y \vee z)=(x \wedge y) \vee(x \wedge z)$ and $(x \vee y) \wedge(x \vee z)=x \vee(y \wedge z)$.

\section{GENERALIZED ALMOST DISTRIBUTIVE FUZZY LATTICES}

In this section, we give some definitions and develop some properties of a Generalized Almost Distributive Fuzzy Lattices. First let us give the definition of an Almost Distributive Fuzzy Lattice

Definition 13: Let $(R, \vee, \wedge, 0)$ be an algebra of type $(2,2,0)$ and $(R, A)$ be a fuzzy poset. Then we call $(R, A)$ is an Almost Distributive Fuzzy Lattice (ADFL) if the following axioms are satisfied:

(F1) $A(a, a \vee 0)=A(a \vee 0, a)=1$,

(F2) $A(0,0 \wedge a)=A(0 \wedge a, 0)=1$,

(F3) $A((a \vee b) \wedge c,(a \wedge c) \vee(b \wedge c))=A((a \wedge c) \vee(b \wedge c),(a \vee$ b) $\wedge c)=1$

(F4) $A(a \wedge(b \vee c),(a \wedge b) \vee(a \wedge c))=A((a \wedge b) \vee(a \wedge c), a \wedge$ $(b \vee c))=1$

(F5) $A(a \vee(b \wedge c),(a \vee b) \wedge(a \vee c))=A((a \vee b) \wedge(a \vee c), a \vee$ $(b \wedge c))=1$

(F6) $A((a \vee b) \wedge b, b)=A(b,(a \vee b) \wedge b)=1$,

for all $a, b, c \in R$.

Definition 14: The fuzzy poset $(R, A)$ is directed above if and only if the poset $(R, \leq)$ is directed above.

Now we give the definition of a Generalized Almost Distributive Fuzzy Lattice as follows:

Definition 15: Let $(R, \vee, \wedge)$ be an algebra of type $(2,2)$ and $(R, A)$ be a fuzzy poset. Then we call $(R, A)$ is a Generalized Almost Distributive Fuzzy Lattice if it satisfies the following axioms:

(FG1) $A((a \wedge b) \wedge c, a \wedge(b \wedge c))=A(a \wedge(b \wedge c),(a \wedge b) \wedge c)=1$;

(FG2) $A(a \wedge(b \vee c),(a \wedge b) \vee(a \wedge c))=A((a \wedge b) \vee(a \wedge c), a \wedge$ $(b \vee c))=1$

(FG3) $A(a \vee(b \wedge c),(a \vee b) \wedge(a \vee c))=A((a \vee b) \wedge(a \vee c), a \vee$ $(b \wedge c))=1$

(FA1) $A(a \wedge(a \vee b), a)=A(a, a \wedge(a \vee b))=1$;

(FA2) $A((a \vee b) \wedge a, a)=A(a,(a \vee b) \wedge a)=1$;

(FA3) $A((a \wedge b) \vee b, b)=A(b,(a \wedge b) \vee b)=1$;

for all $a, b, c \in R$.

Example 16: Let $R=\{a, b, c\}$. Define two binary operations $\vee$ and $\wedge$ on $R$ as follows:

Define a fuzzy relation $A: R \times R \longrightarrow[0,1]$ as follows: $A(a, a)=A(b, b)=A(c, c)=1, A(b, a)=A(b, c)=A(c, a)=$

\begin{tabular}{|l|l|l|l|}
\hline$\vee$ & $a$ & $b$ & $c$ \\
\hline$a$ & $a$ & $a$ & $a$ \\
\hline$b$ & $a$ & $b$ & $b$ \\
\hline$c$ & $c$ & $c$ & $c$ \\
\hline
\end{tabular}$\quad$ and $\quad$\begin{tabular}{|c|c|c|c|}
\hline$\wedge$ & $a$ & $b$ & $c$ \\
\hline$a$ & $a$ & $b$ & $c$ \\
\hline$b$ & $b$ & $b$ & $c$ \\
\hline$c$ & $b$ & $b$ & $c$ \\
\hline
\end{tabular}

$A(c, b)=0, A(a, b)=0.2$ and $A(a, c)=0.4$. Clearly $(R, A)$ is a fuzzy poset. Here $(R, A)$ is a GADFL since it satisfies the above six axioms of a GADFL.

Next, we give an example of a GADFL which is not an ADFL.

Example 17: Let $R=\{a, b, c\}$. Define two binary operations $\vee$ and $\wedge$ on $R$ as follows:

\begin{tabular}{|l|l|l|l|}
\hline$\vee$ & $a$ & $b$ & $c$ \\
\hline$a$ & $a$ & $b$ & $a$ \\
\hline$b$ & $b$ & $b$ & $b$ \\
\hline$c$ & $c$ & $c$ & $c$ \\
\hline
\end{tabular}$\quad$ and $\quad$\begin{tabular}{|c|c|c|c|}
\hline$\wedge$ & $a$ & $b$ & $c$ \\
\hline$a$ & $a$ & $a$ & $c$ \\
\hline$b$ & $a$ & $b$ & $c$ \\
\hline$c$ & $a$ & $a$ & $c$ \\
\hline
\end{tabular}

Define a fuzzy relation $A: R \times R \longrightarrow[0,1]$ as example 3.4 above. Then $(R, A)$ is a GADFL, but it is not an ADFL.

Now, we give some elementary properties of a GADFL. From the definitions of GADL and GADFL, The following theorem is direct.

Theorem 18: Let $(R, A)$ be a fuzzy poset . Then $R$ is a GADL iff $(R, A)$ is a GADFL.

Throughout the remaining of this paper, by $R$ we mean a $\operatorname{GADL}(R, \vee, \wedge)$ unless otherwise specified.

Theorem 19: Let $(R, A)$ be a GADFL . Then $a=b \Leftrightarrow$ $A(a, b)=A(b, a)=1$.

Definition 20: Let $(R, A)$ be a GADFL . Then for any $a, b$ $\in R, a \leq b$ if and only if $A(a, b)>0$.

In view of the above definition, we have the following theorem.

Theorem 21: If $(R, A)$ is a GADFL then $a \wedge b=a$ if and only if $A(a, b)>0$.

Lemma 22: Let $(R, A)$ be a GADFL and $a, b \in R$ such that $a \neq b$. If $A(a, b)>0$ then $A(b, a)=0$.

Lemma 23: Let $(R, A)$ be a GADFL. Then for each $a$ and $b$ in $R$

(1) $A(a \wedge b, b)>0$ and $A(b \wedge a, a)>0$.

(2) $A(a, a \vee b)>0$ and $A(b, b \vee a)>0$.

Lemma 24: Let $(R, A)$ be a GADFL. For any $a \in R$,

(1) $A(a \vee a, a)=1$

(2) $A(a \wedge a, a)=1$.

In addition to the three absorption laws FA1, FA2 and FA3 given in the above definition we also get the following.

Lemma 25: Let $(R, A)$ be a GADFL. For any $a, b \in R$,

(FA4) $A(a \vee(a \wedge b), a)=1$,

(FA5) $A(a \vee(b \wedge a), a)=1$

But a GADFL, in general does not satisfy the following absorption laws.

(FA6) $A((a \vee b) \wedge b, b)=1$,

(FA7) $A((a \wedge b) \vee a, a)=1$,

(FA8) $A(a \wedge(b \vee a), a)=1$

Using the absorption laws FA1 to FA5 the proof of the following lemma is direct.

Lemma 26: Let $(R, A)$ be a GADFL. For any $a, b \in R$, we have 
(1) $A(b, a \wedge b)>0 \Rightarrow A(a \vee b, a)>0$.

(2) $A(a \vee b, b)>0$ and $A(b, a \vee b)>0 \Leftrightarrow A(a, a \wedge b)>0$ and $A(a \wedge b, a)>0$.

Notice that in a GADFL the converse of above lemma does not hold true, i.e $A(a \vee b, a)>0$ need not imply $A(b, a \wedge b)>0$. Since in example 3.5 we get $A(c \vee b, c)=A(c, c)=1>0$. But $A(b, c \wedge b)=A(b, a)=0$ which is not greater than 0 . Using the absorption laws FA1 through FA5 and the above lemma, we have the following corollary:

Corollary 27: Let $(R, A)$ be a GADFL. For any $a, b \in R$, we have

(1) $A(a \vee(a \vee b), a \vee b)=1$;

(2) $A((a \vee b) \vee a, a \vee b)=1$;

(3) $A(a \wedge(b \wedge a), b \wedge a)=1$.

We have already obtained certain absorption laws that a GADFL satisfies in general. Regarding the remaining absorption laws we have the following theorem.

Theorem 28: Let $(R, A)$ be a GADFL. For any $a, b \in R$, the following are equivalent:

(1) $A(a \wedge(b \vee a), a)>0$ and $A(a, a \wedge(b \vee a))>0$;

(2) $A((a \wedge b) \vee a, a)>0$ and $A(a,(a \wedge b) \vee a)>0$;

(3) $A(b \wedge(a \vee b), b)>0$ and $A(b, b \wedge(a \vee b))>0$;

(4) $A((b \wedge a) \vee b, b)>0$ and $A(b,(b \wedge a) \vee b)>0$;

(5) $A(a \wedge b, b \wedge a)>0$ and $A(b \wedge a, a \wedge b)>0$;

(6) $A(a \vee b, b \vee a)>0$ and $A(b \vee a, a \vee b)>0$.

Proof: Let $(R, A)$ be a GADFL and $a, b \in R$

(1) $\Leftrightarrow(2)$

$$
\begin{aligned}
A(a \wedge(b \vee a), a)>0 & \Leftrightarrow A((a \wedge b) \vee(a \wedge a), a)>0 \\
& \Leftrightarrow A((a \wedge b) \vee a, a)>0
\end{aligned}
$$

and

$$
\begin{aligned}
A(a, a \wedge(b \vee a))>0 & \Leftrightarrow A(a,(a \wedge b) \vee(a \wedge a))>0 \\
& \Leftrightarrow A(a,(a \wedge b) \vee a)>0
\end{aligned}
$$

Hence (1) and (2) are equivalent.

By interchanging the roles of $a$ and $b$ we get the equivalence of (3) and (4).

$(2) \Leftrightarrow(5):$ Suppose (2)

$A(a \wedge b, b \wedge a)=A(a \wedge b, b \wedge\{(a \wedge b) \vee a\})=A(a \wedge b,\{b \wedge(a \wedge$ $b)\} \vee(b \wedge a))=A(a \wedge b,(a \wedge b) \vee(b \wedge a))=A(a \wedge b,(a \wedge b) \vee$ $\{a \wedge(b \wedge a)\})=A(a \wedge b, a \wedge(b \vee(b \wedge a)))=A(a \wedge b, a \wedge b)=1$. $A(a \wedge b, b \wedge a)>0$. Similarly, $A(b \wedge a, a \wedge b)>0$.

Suppose (5)

$A((a \wedge b) \vee a, a)=A((b \wedge a) \vee a, a)=1$. Hence $A((a \wedge b) \vee a, a)>0$. Similarly, $A(a,(a \wedge b) \vee a)>0$. Therefore (2) and (5) are equivalent. By interchanging the roles of $a$ and $b$, we get $(4) \Leftrightarrow(5)$.

Thus (1) to (5) are equivalent. We complete the proof by proving the equivalence of (6) and (1). (6) $\Rightarrow(1)$ is direct. Suppose (1) $A(a \vee b, b \vee a)=A(a \vee b, b \vee\{(a \vee b) \wedge a\})=$ $A(a \vee b,\{b \vee(a \vee b)\} \wedge(b \vee a))=A(a \vee b,(a \vee b) \wedge(b \vee a))=$ $A(a \vee b,(a \vee b) \wedge\{a \vee(b \vee a)\})=A(a \vee b, a \vee\{b \wedge(b \vee a)\})=$ $A(a \vee b, a \vee b)=1$.

Hence $A(a \vee b, b \vee a)>0$. Similarly $A(b \vee a, a \vee b)>0$.

Definition 29: Let $(R, A)$ be a GADFL. For any $a, b \in R$ we say that $a$ is less than or equal to $b$ and write $a \leq b$ if $A(a \wedge$ $b, a)>0$ and $A(a, a \wedge b)>0$ or, equivalently, $A(a \vee b, b)>0$ and $A(b, a \vee b)>0$.

Lemma 30: Let $(R, A)$ be a GADFL. If $a, b \in R$ such that $a \leq b$ then ;

(1) $A(a \wedge b, b \wedge a)>0$ and $A(b \wedge a, a \wedge b)>0$;

(2) $A(a \wedge(b \vee a), a)=1$

(3) $A(a \vee b, b \vee a)=1$

Now we give the equivalent conditions of commutativity of join for any two elements in $R$.

Theorem 31: Let $(R, A)$ be a GADFL. For any $a, b \in R$, the following are equivalent;

(1) $A(a \vee b, b \vee a)>0$ and $A(b \vee a, a \vee b)>0$;

(2) $A(b, a \vee b)>0$;

(3) There exists $c \in R$ such that $A(a, c)>0$ and $A(b, c)>0$;

(4) $a \vee b$ is the lub of $a$ and $b$ in $R$;

(5) $A(a, b \vee a)>0$;

(6) $b \vee a$ is the lub of $a$ and $b$ in $R$.

In the following, we give the equivalent conditions of commutativity of meet for any two elements in $R$.

Theorem 32: Let $(R, A)$ be a GADFL. For any $a, b \in R$, the following statements are equivalent.

(1) $A(a \wedge b, b \wedge a)>0$ and $A(b \wedge a, a \wedge b)>0$;

(2) $A(a \wedge b, a)>0$;

(3) $a \wedge b$ is the glb of $a$ and $b$ in $R$;

(4) $A(b \wedge a, b)>0$;

(5) $b \wedge a$ is the glb of $a$ and $b$ in $R$.

Since in a GADL we know that $a \wedge b \wedge c=b \wedge a \wedge c$ for any $a, b, c \in R$ we have the following lemma.

Lemma 33: Let $(R, A)$ be a GADFL. For any $a, b, c \in R$ $A(a \wedge b \wedge c, b \wedge a \wedge c)=1$.

Now, we give the following conditions for a GADFL to become a distributive fuzzy lattice.

Theorem 34: Let $(R, A)$ be a GADFL. Then the following are equivalent.

(1) $(R, A)$ is distributive fuzzy lattice;

(2) The fuzzy poset $(R, A)$ is directed above;

(3) $A(a \wedge(b \vee a), a)>0$ and $A(a, a \wedge(b \vee a))>0$;

(4) $A(a \vee b, b \vee a)>0$ and $A(b \vee a, a \vee b)>0$;

(5) $A(a \wedge b, b \wedge a)>0$ and $A(b \wedge a, a \wedge b)>0$;

(6) The relation $\theta=\{(a, b) \in R \times R \mid A(b, a \wedge b)>0\}$ is antisymmetric.

Proof: Let $(R, A)$ be a GADFL and $a, b \in R$, then

(1) $\Rightarrow(2)$

Suppose $(R, A)$ is distributive fuzzy lattice. For any $a, b \in R$ we have $A(a, a \vee b)>0$ and $A(b, a \vee b)>0$. Take $c=a \vee b$. Then $A(a, c)>0$ and $A(b, c)>0 \Rightarrow a \leq c$ and $b \leq c$ for $c \in R$. $\Rightarrow$ $R$ is directed above. Hence the fuzzy poset $(R, A)$ is directed above. The equivalence of (2), (4), (5) and (3) follows from Theorem 31 and Theorem 34

$(5) \Rightarrow(6)$

Suppose $(a, b) \in \theta$ and $(b, a) \in \theta$

Monte Carlo: $a=b$

$(a, b) \in \theta \Rightarrow a \wedge b=b$

$(b, a) \in \theta \Rightarrow b \wedge a=a$.

Because of (5) and antisymmetric property of $A$ we have $a \wedge b=b \wedge a$ and hence $a=b$.

(6) $\Rightarrow(1)$ 
Suppose $\theta=\{(a, b) \in R \times R \mid A(b, a \wedge b)>0\}$ is antisymmetric. Since $a \wedge b \wedge b \wedge a=b \wedge a \Rightarrow(a \wedge b, b \wedge a) \in \theta$.

Similarly, $b \wedge a \wedge a \wedge b=a \wedge b \Rightarrow(b \wedge a, a \wedge b) \in \theta$.

Hence by antisymmetry property of $\theta a \wedge b=b \wedge a$.

$\Rightarrow A(a \wedge b, b \wedge a)>0$ and $A(b \wedge a, a \wedge b)>0$.

Now, by theorems $3.15((5) \Leftrightarrow(6)), 35((1) \Leftrightarrow(4))$ and $3.20((1)$ $\Leftrightarrow(3))$ we have $(R, A)$ is a fuzzy poset in which for any $a, b \in$ $R, a \wedge b$ is glb of $a$ and $b, a \vee b$ is lub of $a$ and $b$ and $\vee$ and $\wedge$ are commutative.

Hence $(R, A)$ is distributive fuzzy lattice.

Finally, we conclude this section with the following theorem which gives the equivalent conditions for a GADFL to become an ADFL.

Theorem 35: Let $(\mathrm{R}, \mathrm{A})$ be a GADFL. Then the following are equivalent.

(1) $(R, A)$ is an Almost Distributive Fuzzy Lattice;

(2) $A((a \vee b) \wedge c,(a \wedge c) \vee(b \wedge c))>0$ and $A((a \wedge c) \vee(b \wedge c),(a \vee b) \wedge c)>0$

(3) $A((a \vee b) \wedge b, b)>0$ and $A(b,(a \vee b) \wedge b)>0$;

(4) $A((a \vee b) \wedge c,(b \vee a) \wedge c)>0$ and

$A((b \vee a) \wedge c,(a \vee b) \wedge c)>0$

for all $a, b, c \in R$.

Proof: Let $(R, A)$ be a GADFL and $a, b, c \in R$

(1) $\Rightarrow(2)$

Suppose $(R, A)$ is an Almost Distributive Fuzzy Lattice. Then we have,

$A((a \vee b) \wedge c,(a \wedge c) \vee(b \wedge c))=A((a \wedge c) \vee(b \wedge c),(a \vee b) \wedge$ $c)=1$.

$\Rightarrow A((a \vee b) \wedge c,(a \wedge c) \vee(b \wedge c))>0$ and $A((a \wedge c) \vee(b \wedge$ c), $(a \vee b) \wedge c)>0$.

Hence (2) holds true.

(2) $\Rightarrow$ (3): Suppose (2)

$A((a \vee b) \wedge b, b)=A((a \wedge b) \vee(b \wedge b), b)=A((a \wedge b) \vee b, b)=1$. Hence $A((a \vee b) \wedge b, b)>0$. Similarly, $A(b,(a \vee b) \wedge b)>0$.

(3) $\Rightarrow(4)$

Since $((a \vee b) \wedge c) \vee c=c$ and $((b \vee a) \wedge c) \vee c=c$.

We have $(a \vee b) \wedge c \leq c$ and $(b \vee a) \wedge c \leq c$

$\Rightarrow A((a \vee b) \wedge c, c)>0$ and $A((b \vee a) \wedge c, c)>0$.

Hence by theorems $3.19((3) \Leftrightarrow(4))$ and $3.16((5) \Leftrightarrow(6))$

we have

$A([(a \vee b) \wedge c] \wedge[(b \vee a) \wedge c],[(b \vee a) \wedge c] \wedge[(a \vee b) \wedge c])>0$ and $A([(b \vee a) \wedge c] \wedge[(a \vee b) \wedge c],[(a \vee b) \wedge c] \wedge[(b \vee a) \wedge c])>0$

$\Rightarrow A((a \vee b) \wedge[c \wedge((b \vee a) \wedge c)],(b \vee a) \wedge[c \wedge((a \vee b) \wedge c)])>0$ and

$A((b \vee a) \wedge[c \wedge((a \vee b) \wedge c)],(a \vee b) \wedge[c \wedge((b \vee a) \wedge c)])>0$

$\Rightarrow A((a \vee b) \wedge(b \vee a) \wedge c,(b \vee a) \wedge(a \vee b) \wedge c)>0$ and

$A((b \vee a) \wedge(a \vee b) \wedge c,(a \vee b) \wedge(b \vee a) \wedge c)>0$

$\Rightarrow A(([(a \vee b) \wedge b] \vee[(a \vee b) \wedge a]) \wedge c,([(b \vee a) \wedge a] \vee[(b \vee$

$a) \wedge b]) \wedge c)>0$ and $A(([(b \vee a) \wedge a] \vee[(b \vee a) \wedge b]) \wedge c$, $([(a \vee b) \wedge b] \vee[(a \vee b) \wedge a]) \wedge c)>0$

$\Rightarrow A((b \vee a) \wedge c,(a \vee b) \wedge c)>0$ and $A((a \vee b) \wedge c,(b \vee a) \wedge c)>$ 0

Hence (4) holds true.

(4) $\Rightarrow(1)$ : Suppose (4)

$A((a \vee b) \wedge c,(a \wedge c) \vee(b \wedge c))=A((a \vee b) \wedge c,[(a \wedge c) \vee b] \wedge[(a \wedge$ $c) \vee c])=A((a \vee b) \wedge c,[(a \wedge c) \vee b] \wedge c)=A((a \vee b) \wedge c,[b \vee$ $(a \wedge c)] \wedge c)=A((a \vee b) \wedge c,[(b \vee a) \wedge(b \vee c)] \wedge c)=A((a \vee$ $b) \wedge c,([(b \vee a) \wedge b] \vee[(b \vee a) \wedge c]) \wedge c)=A((a \vee b) \wedge c,([(b \vee$ $a) \wedge c] \vee[(b \vee a) \wedge b]) \wedge c)=A((a \vee b) \wedge c,[(b \vee a) \wedge(c \vee b)] \wedge c)$ $=A((a \vee b) \wedge c,(b \vee a) \wedge[(c \vee b) \wedge c])=A((a \vee b) \wedge c,(b \vee a) \wedge c)$ $=A((a \vee b) \wedge c,(a \vee b) \wedge c)=1$.

Hence $A((a \vee b) \wedge c,(a \wedge c) \vee(b \wedge c))=1$. Similarly, $A((a \wedge c) \vee$ $(b \wedge c),(a \vee b) \wedge c)=1$. Therefore $A((a \vee b) \wedge c,(a \wedge c) \vee(b \wedge$ $c))=A((a \wedge c) \vee(b \wedge c),(a \vee b) \wedge c)=1$. Also, $A((a \vee b) \wedge b, b)$ $=A(b,(a \vee b) \wedge b)=1$. Hence $(R, A)$ is an Almost Distributive Fuzzy Lattice.

\section{REFERENCES}

[1] G. Rao, R. Bandaru, and N. Rafi, "Generalized almost distributive latticesi," Southeast Asian Bulletin of Mathematics, vol. 33, no. 6, 2009.

[2] U. Swamy and G. Rao, "Almost distributive lattices," Journal of the Australian Mathematical Society (Series A), vol. 31, no. 1, pp. 77-91, 1981.

[3] L. Zadeh, "Fuzzy sets," Information and control, vol. 8, no. 3, pp. 338353, 1965.

[4] - "Similarity relations and fuzzy orderings," Information sciences, vol. 3, no. 2, pp. 177-200, 1971.

[5] N. Ajmal and K. Thomas, "Fuzzy lattices," Information sciences, vol. 79, no. 3-4, pp. 271-291, 1994.

[6] I. Chon, "Fuzzy partial order relations and fuzzy lattices," Korean J. Math, vol. 17, no. 4, pp. 361-374, 2009. 